\title{
Significance of numerical chromosome abnormalities in children with acute leukaemias detected by flow cytometry
}

\author{
Magdalena Pierzyna-Świtała, Łukasz Sędek, Bogdan Mazur \\ Department of Microbiology and Immunology, Medical University of Silesia in Katowice, Zabrze, Poland
}

ABSTRACT

Acute leukaemias (AL) constitute a heterogeneous group of diseases that differ in terms of immunophenotype, as well as structural and numerical chromosome alterations. In recent years research has revealed many genetic abnormalities in leukaemic cells. Some of them have already well-grounded diagnostic and prognostic significance in acute lymphoblastic leukaemia (ALL) and play an important role in patient risk stratification. Besides cytogenetics, which still remains a gold standard for the classification of AL, also flow cytometry can be useful in detecting abnormal numbers of chromosomes in leukemic blasts. The presence of DNA aneuploidy may be an early indicator of cytogenetic abnormality directly associated with response to therapy. High hyperdiploidy with 51-65 chromosomes and DNA index > 1.16 is a favourable prognostic factor in ALL in children, often related to CD123 antigen overexpression. The aim of this study was to summarise current knowledge about numerical chromosome abnormalities in AL diagnosed by flow cytometry.

KEY WORDS:

acute leukaemia, flow cytometry, aneuploidy, DNA index, ploidy.

\section{INTRODUCTION}

Acute leukaemias (AL) constitute a heterogeneous group of diseases that differ in terms of immunophenotype as well as structural and numerical chromosome alterations. AL is a malignant disease of the bone marrow, in which early lymphoid or myeloid precursors proliferate. As a consequence, normal haematopoietic cells of the marrow are replaced with leukaemic cells (blasts). Acute lymphoblastic leukaemia (ALL) is the most common type of leukaemia in children between two and four years of age, while acute myeloid leukaemia (AML) occurs more frequently in adults above the age of 40 years and it is rare in children [1]. The first classification system of leukaemias - the French-American-British (FAB) classification, was based on morphological features of leukaemic cells. In this classification, ALL was divided into three (L1-L3), and AML into eight (M0-M7), morphological subtypes [2]. This classification system was later supplemented with immunophenotypic features of leukaemic blasts assessed by flow cytometry, which was the basis of the European Group for the Immunological Characterisation of Leukaemias (EGIL) classification of ALL [3]. More recently, the World Health Organisation (WHO) created in 2008 a new classification implementing cytogenetics, genetics, and immunophenotypic features of leukaemic blasts [4]. The WHO classification is systematically updated, based on new research findings [5]. Leukaemic blasts of all known AL types often bear some chromosomal abnormalities concerning numerical and/or structural alterations. Some of them can have favourable or unfavourable clinical meaning, which will be reviewed in the

\section{ADDRESS FOR CORRESPONDENCE:}

Magdalena Pierzyna-Świtała, Department of Microbiology and Immunology, Medical University of Silesia in Katowice, 19 Jordana St., 41-808 Zabrze, Poland, e-mail: magdalena.pierzyna@gmail.com 
following sections. Importantly, chromosomal abnormalities are included in the list of factors determining proper risk stratification for children with ALL [6].

Chromosomal instability manifested as DNA aneuploidy is considered as an early step to leukaemogenesis [6]. Conventional cytogenetic analysis still remains the gold standard to detect chromosome abnormalities in haematological malignancies. However, new techniques are being used to confirm the cytogenetic findings or to define the risk group when cytogenetics is not available or inconclusive [7]. In a large percentage of patients with $\mathrm{AL}$, undetected aneuploidy could exist and may not be identified by conventional cytogenetics at the time of diagnosis. Some discrete chromosomal changes can only be detected with more sensitive techniques, such as fluorescence in situ hybridisation (FISH) or flow cytometry (FC). The aim of this study was to summarise current knowledge about numerical chromosome abnormalities in AL detected by flow cytometry and their clinical significance.

\section{TECHNIQUES FOR DETECTING NUMERICAL CHROMOSOME ABNORMALITIES}

Chromosome analysis has become an important diagnostic tool in the assessment of patients with AL. There are a few techniques than can be utilised to detect chromosome abnormalities in haematological malignancies.

\section{CONVENTIONAL CYTOGENETICS}

Conventional cytogenetics (CCA) is commonly used as a routine diagnostic test to detect chromosome abnormalities in haematological malignancies. It can identify all chromosome aberrations visible under the microscope in a leukaemic cell. The methodology is based on karyotyping of cultured bone marrow samples from patients with leukaemias, and producing G-banded metaphases for microscopic analysis [8]. CCA of patients with ALL is often difficult due to the poor quality of chromosomes in metaphase of mitotic division, limited number of spread metaphase chromosomes, or poor chromosome morphology [9]. At least 20 metaphases must be examined to exclude clonal abnormality. In cases involving clonal aberrancies, any detected chromosomal aberration (such as extra chromosomes, structural aberrations) must be confirmed in a minimum of two cells, or three cells in cases of chromosome loss. However, it is not clear if the abnormalities detected in a few analysable metaphases under optimal karyotyping conditions are representative for whole leukaemic cells [10].

\section{FLUORESCENCE IN SITU HYBRIDISATION}

Many chromosome aberrations recurrent in leukaemic cells can be detected using molecular techniques, such as FISH. It is a powerful tool for the identification of numerical aberrations of the targeted chromosomes in metaphases and interphase cells [9]. Ritterbach et al. [9] suggested that by using centromeric probes for chromosomes $6,10,17$, and 18 it is possible to detect hyperdiploidy $>50$ chromosomes, which can be useful for therapy stratification. This is an alternative method to screen for hyperdiploidy allowing for detection of even small clones, as compared with classical cytogenetics. FISH provides a sensitive method for identification of chromosomal translocations in metaphase, as well as interphase cells, and can be reliably performed on diagnostic bone marrow smears. However, the disadvantage of this method is the high cost [1].

\section{FLOW CYTOMETRY}

During recent years, DNA aneuploidy determined by FC was a widely used parameter in the prognostication of many solid tumours and haematological malignancies [11-13]. Nowadays, flow cytometry FC is routinely used for rapid assessment of the immunophenotype of leukaemic cells, and minimal residual disease (MRD) detection enables also the assessment of the ploidy of leukemic blasts and measurement of DNA content in parallel. Flow cytometric ploidy assessment is based on total cellular DNA evaluation and does not identify individual chromosomes; however, it has been reported that DNA index (DI) values can be used indirectly to determine the number of chromosomes $[6,14]$. The measurement of DNA content is based on the ability of certain dyes (such as propidium iodide - PI) to stain cellular DNA in a stoichiometric manner. The amount of bound PI is proportional to the amount of DNA in the cell, which can give information on possible changes in the number of chromosomes [8]. The results are presented graphically as a histogram, and the difference in DNA content can be expressed as a ratio of leukaemia sample/standard DNA fluorescence, defined as DI.

FC is a rapid, informative, sensitive method for measurement of DNA content and can be applied to a wide range of disorders [8]. FC enables detection of even small aneuploid clones and ensures faster identification of abnormal DNA content. The great advantage of FC is its capability of analysis of high numbers of cells in comparison to other methods. It also enables identification of ploidy variations in the majority of ALL patients. However, in routine practice it cannot replace conventional cytogenetics and typically is used in parallel as a supplementary technique. FC allows the identification of biclonality and distinguishes aneuploid leukaemia when standard CCA fails [15]. Importantly, the comparison between the FISH result and the DI showed good agreement in the investigated patients $(208 / 234,88.8 \%)$ [9]. Forestier et al. [15] suggested that DI assessment is a sensitive and reliable method to identify hyperdiploid ALL. Moreover, Kenney et al. suggested that DNA ploidy in follow-up can 
improve detection of relapses in B-ALL [16]. A proposal for a new strategy algorithm for ALL was presented by Pérez-Vera et al. [10] in order to provide a more accurate diagnosis and better prognostic stratification of children with chromosomal abnormalities. To improve the frequency of chromosome abnormality detection they performed a study on 100 newly diagnosed ALL children and compared with the numerical abnormalities identified by different methods. The concordance between conventional cytogenetics and flow cytometry was $86 \%$. Therefore, they suggest, based on their results, that for numerical aberration detection, the flow cytometry technique should be used first. Then molecular testing should be introduced to give the oncologist more information to assess the patient's risk. This strategy may allow the detection of numerical and structural abnormalities in a few days.

\section{NUMERICAL CHROMOSOME ABNORMALITIES IN B-CELL PRECURSOR ACUTE LYMPHOBLASTIC LEUKAEMIA}

The most frequent chromosomal abnormalities found in B-cell precursor acute lymphoblastic leukaemia (BCPALL) are high hyperdiploidy (51-65 chromosomes) and the translocation $\mathrm{t}(12 ; 21)(\mathrm{p} 13 ; \mathrm{q} 22)$ resulting in ETV6RUNX1 fusion gene formation. These two aberrancies comprise together more than $50 \%$ of cases and are at the same time markers of good prognosis. Other abnormalities, for example the translocation $\mathrm{t}(9 ; 22)$ (q34;q11) with fusion gene product $B C R-A B L 1$, as well as rearrangements of the $M L L$ gene, are rare in childhood BCP-ALL [17], but both are related to poor prognosis, especially the $M L L$ gene rearrangements in infant ALL, which is associated with an aggressive disease and unfavourable outcome [18]. In contrast, hypodiploidy ( $<46$ chromosomes) is a marker of poor outcome; the fewer chromosomes the worse the prognosis $[19,20]$.

ALL are classified into subtypes based on the prevalent chromosome number in leukaemic cells, defined as the modal number (MN) of chromosomes. About 25-30\% of BCP-ALL cases are hyperdiploid with more than 50 chromosomes. This subtype of ALL includes near-tetraploid, near-triploid, and hyperdiploid with 5168 chromosomes. Moderate hyperdiploidy (47-50 chromosomes) occurs in 10 to $15 \%$ of ALL childhood leukaemia. Diploid with 46 chromosomes and pseudodiploid BCP-ALL with 46 chromosomes and structural and/ or numerical abnormalities occur in $40 \%$ and $10-15 \%$ of cases, respectively. Fewer than 46 chromosomes are typical for hypodiploidy, and, together with near-haploidy cases, this occurs altogether in about $8 \%$ of cases. Recognition of ploidy in ALL has improved the ability to predict clinical outcome and devise risk-specific

TABLE 1. Numeric chromosomal aberrations in B-cell precursor acute lymphoblastic leukaemia (BCP-ALL)

\begin{tabular}{|c|c|c|c|c|c|}
\hline & & MN & Outcomes & Characteristic & Immunophenotype \\
\hline \multirow{4}{*}{$\begin{array}{l}\text { 흥 } \\
\text { 응 } \\
\text { ㅎ⿺ㄴ } \\
\text { 종 }\end{array}$} & $\begin{array}{l}\text { Near- } \\
\text { tetraploidy }\end{array}$ & $82-94$ & unfavourable & $\begin{array}{l}\text { associated with } L 2 \text { morphology, T-cell } \\
\text { immunophenotype, older age at the time } \\
\text { of diagnosis }\end{array}$ & $\begin{array}{l}\text { myeloid-associated antigens CD13, CD15, } \\
\text { and CD33 }\end{array}$ \\
\hline & Near-triploidy & $66-73$ & favourable & $\begin{array}{l}\text { associated with pre-B-cell immunopheno- } \\
\text { type }\end{array}$ & ND \\
\hline & $\begin{array}{l}\text { High } \\
\text { hyperdiploidy }\end{array}$ & $51-68$ & favourable & $\begin{array}{l}\text { associated with low white cell count, L1 or } \\
\text { L2 morphology; gain X, 4, } 6,10,14,17,18 \text {, } \\
\text { and } 21 \text { chromosome; also characterised by } \\
\text { lower leukocyte counts, lower serum lactic } \\
\text { dehydrogenase levels, ages between } 2 \text { and } \\
10 \text { years; allelic variants of PRDM9 }\end{array}$ & $\begin{array}{l}\text { the presence of common ALL immu- } \\
\text { nophenotype; associated with CD10q } \\
\text { overexpression, CD66c positivity and the } \\
\text { absence of surface CD45; CD123 } \\
\text { (IL-3 receptor a chain) overexpress in } \\
\text { B-precursor ALL with hyperdiploid cases } \\
\text { with karyotype } \geq 52 \text { chromosomes }\end{array}$ \\
\hline & $\begin{array}{l}\text { Low } \\
\text { hyperdiploidy }\end{array}$ & $47-50$ & intermediate & $\begin{array}{l}\text { the most frequently acquired numerical } \\
\text { abnormalities were } 21, X, 8 \text { and } 10\end{array}$ & ND \\
\hline \multirow{3}{*}{$\begin{array}{l}\text { 흥 } \\
\frac{0}{\overline{0}} \\
\text { 을 } \\
\text { 조 }\end{array}$} & $\begin{array}{l}\text { High } \\
\text { hypodiploidy }\end{array}$ & $42-45$ & unfavourable & $\begin{array}{l}\text { complex karyotypes involving chromosomes } \\
7,9,12\end{array}$ & $\begin{array}{l}\text { included patients with T-ALL } \\
\text { and pre-B/common immunophenotype }\end{array}$ \\
\hline & $\begin{array}{l}\text { Low } \\
\text { hypodiploidy }\end{array}$ & $33-39$ & unfavourable & $\begin{array}{l}\text { monosomy of chromosomes } 3,7,15,16,17 \\
\text { and disomy of chromosomes 1, 6, 11, 18; } \\
\text { correlation with somatic TP53 mutations; } \\
\text { restricted to children of median age } 15 \text { years }\end{array}$ & common/pre-B immunophenotype \\
\hline & Near-haploidy & $23-29$ & unfavourable & $\begin{array}{l}\text { correlation with mutations in IKZF3 and } \\
\text { FLT3; restricted to children of median age } \\
7 \text { years; low WBC count }\end{array}$ & common/pre-B immunophenotype \\
\hline
\end{tabular}

$M N$ - modal number, $N D$ - no data 
therapy [20]. Numeric chromosomal aberrations and their outcomes in BCP-ALL are presented in Table 1.

\section{HYPERDIPLOIDY}

Near-tetraploidy (MN: 82 to 94 chromosomes) occurs in less than $1 \%$ of childhood ALL. According to literature, near-tetraploidy in $30 \%$ of cases appears to be associated with L2 morphology, in 47\% with T-cell immunophenotype and older age at diagnosis. Moreover, $40 \%$ of near-tetraploid cases express at least one myeloid-associated antigen CD13, CD15, and CD33. Most of these patients achieve remission in a short time. However, Pui et al. suggested that this subgroup should be considered as a district entity from the other hyperdiploid cases because the follow-up of the majority of patients who remain in remission is shorter. Thus, near-tetraploid ALL should be separated from hyperdiploidy $>50$ cases, which have a better prognosis [21].

Near-triploid karyotype (MN: 66 to 73 chromosomes) is a rare finding in childhood leukaemia and is associated more often with pre-B-cell immunophenotype according to the EGIL classification of BCP-ALL [21].

High hyperdiploidy karyotype (51-65 chromosomes) occurs in 25 to $30 \%$ of childhood leukaemia, and this group of patients has more favourable prognosis than others. It is characterised by the gain of chromosomes $4,6,10$, $14,17,18,21$, and X. Extra copies of chromosome 4, 6, 10, 14 , and 18 more often result in trisomy than tetrasomy. Other trisomies and tetrasomies are also occasionally seen $[22,23]$. Moreover, this group can also be characterised by lower leukocyte counts, lower serum lactate dehydrogenase levels, ages between 2 and 10 years, and the presence of common ALL immunophenotype [24]. Moorman et al. [25] confirmed that the outcomes of children younger than 10 years with high hyperdiploid are heterogeneous, and trisomies of both chromosome 4 and 18 can identify patients with better prognosis, with a five-year event-free survival of $96 \%$ vs. $84 \%$ at five years, $p<0.0001$. High hyperdiploidy was also used for risk stratification by The Children's Oncology Group (COG) because trisomy of chromosomes 4,10 , and 17 was associated with good prognosis [26]. Recently, Woorward et al. [27] reported that rare allelic variants of PRDM9 are associated with high hyperdiploidy childhood ALL.

Zaliova et al. [24] demonstrated that cytogenetically defined high hyperdiploidy can be divided into two subgroups with different biological behaviour. A relevant cut-off value of DI was set to DI $=1.16$. As confirmed with single nucleotide polymorphism array, patients with DI $>1.16$ had a better survival rate than patents with DI $<1.16$ [28]. Moreover, better response in the early treatment phases in patients with higher DI was associated with trisomy of chromosomes 4, 6, 10, 22 or combined trisomies $4+10,4+17$, or $4+10+17$. In contrast, trisomy 5 was referred as a negative prognostic feature [24]. On the other hand, the low hyperdiploid subgroup with 47-50 chromosomes had worse outcome than high hyperdiploid DI $<1.16$ [24]. In this group, the most frequently acquired numerical abnormalities was the gain of chromosome 21, X, 8, and 10 [29].

High hyperdiploid ALL is frequently associated with CD10 overexpression, CD66c positivity, and the absence of surface CD45 [30]. Immunophenotyping at ALL diagnosis shows also higher rates of CD123 (IL-3 receptor a chain) overexpression ( $81 \%$ of BCP-ALL with hyperdiploidy and karyotype $\geq 52$ chromosomes). Therefore, CD123 seems to constitute an additional marker of good prognosis in childhood ALL. Moreover, CD123 showed stable overexpression in $77 \%$ of patients at follow-up [31], particularly because expression of this antigen is not observed on normal lymphoid precursors [32].

\section{HYPODIPLOIDY}

Hypodiploidy (< 46 chromosomes) in childhood ALL is associated with a very high risk of treatment failure [33]. Hypodiploid ALL with fewer than 46 chromosomes occurs in $1 \%$ of childhood ALL and may be subdivided depending on MN into high hypodiploidy (42-45 chromosomes), low hypodiploidy (33-39 chromosomes), and near-haploidy (23-29 chromosomes) subgroups [33, 34]. Most hypodiploid cases have chromosomal deficiency arising from unbalanced translocation, loss of whole chromosome, or formation of dicentric chromosomes. Raimondi et al. [35] detected loss of sex chromosomes in $63 \%$ of cases with hypodiploidy. Heerema et al. [36] suggested that patients with decreased chromosome number had worse outcomes and that this subset of patients should receive different treatment protocols. Both near-haploidy and low hypodiploid tumours exhibit distinct mutational profiles, suggesting possible usage of drugs directed against Ras- and PI3k-dependent signalling pathways as new therapeutic strategy [37]. Occasionally, in hypodiploid cases, chromosomes can undergo reduplication and production of so-called double-up subclone in a mechanism called "masked hypodiploid ALL". This results in a karyotype resembling hyperdiploidy and can cause classification of patients into incorrect risk groups [19, 34, 38].

In near-haploid ALL cases, leukaemic blasts contain 23 to 29 chromosomes, and the incidence of near-haploidy is about $1 \%$. Among this group the majority of patients have 26 chromosomes. According to Harrison et al. the most frequently observed additions to the haploid chromosome set are extra chromosome 21, observed in all patients, X (6/8 females), Y (4/6 males), 14 (12/226 cases), and 18 (7/226 cases). Moreover, near-haploidy is often restricted to children of median age seven years (range two to 15 years). The majority of patients have common-B or pre-B immunophenotype [33]. This group of aneuploidies 
is poorly defined; however, earlier studies reported poor outcomes for children with near-haploidy $[35,39,40]$.

Low hypodiploidy (33-39 chromosomes) ALL is recognised as a high-risk feature in paediatric ALL. In this subgroup, monosomy of chromosomes 7 and 17 are always observed. Other frequently observed additions to the haploid set of chromosomes are chromosomes 21, 14, 18 , and $\mathrm{X}$ in females and $\mathrm{Y}$ in males. All hypodiploid ALL patients have common-B or pre-B immunophenotypes. This subtype occurred in an older group with a median age of 15 years (range nine to 54 years) [33]. Both high and low hypodiploidy, are associated with somatic TP53 mutations, which suggests the existence of similar mutational patterns in both subtypes of hypodiploid ALL [34].

High hypodiploidy (42-45 chromosomes) are clinically and cytogenetically distinct from the near-haploid and low hypodiploid group. Harrison et al. [33] reported complex karyotypes involving chromosomes 7, 9, and 12 in the majority of high hypodiploidy patients (95\%). Loss of whole chromosomes: sex, 7, 9, 13, and 17 was also often detected.

\section{NUMERICAL CHROMOSOME ABNORMALITIES IN T-ALL}

The impact of numerical chromosome abnormalities on T-ALL prognosis is less well understood than in BCP-ALL [35]. Near-haploidy and hyperdiploidy above 50 chromosomes are very rare in paediatric T-ALL. Near-tetraploidy occurs in about $1-3 \%$ of T-ALL, but no prognostic significance has been determined [41, 42]. Pui et al. [21] reported that cases of near-tetraploidy occurring in ALL are often associated with T-ALL immunophenotype (47\%), L2 morphology (30\%), older age at diagnosis, and poorer prognosis as compared to cases with other types of ploidy. In contrast, the EGIL group collected data on 36 children with near-tetraploidy (81-103 chromosomes). In this study 10 cases (48\%) showed T-ALL immunophenotype and DI determined for 21 patients ranging between 1.74 and 2.08. In general, the overall survival of this patient subgroup appeared to be favourable. All blasts were negative for HLA-DR and 6/7 were negative for CD34. Expression for cyCD79a was found in 4/5 tested samples and TdT in 5/5 tested samples. Also, expression of myeloid-associated antigens was found (CD13, CD33, and CD117 for 2/6, 2/7, and 2/4 examined cases, respectively) confirming high immunophenotypic heterogeneity of near-tetraploid cases [43]. Another study of Munoz et al. [32] proved that none of six hyperdiploid T-ALL cases expressed CD123 antigen, which is in contrast to BCP-ALL.

\section{NUMERICAL CHROMOSOME ABNORMALITIES IN ACUTE MYELOID LEUKAEMIA}

In patients with $\mathrm{AML}$, some genetic abnormalities, like translocations $\mathrm{t}(8 ; 21), \mathrm{t}(15 ; 17), \mathrm{t}(16 ; 16)$, or inv(16) are considered as markers of favourable outcome. In contrast, abnormalities in chromosomes 5 or $7,11 \mathrm{q} 23$, or complex karyotypes are associated with very poor outcome, regardless of the treatment protocol used [1]. Only a few reports are available about ploidy in AML. Generally, the frequency of abnormalities in children is higher as compared with adult AML [44]. One of the largest series of paediatric AML patients $(n=237)$ with annotated ploidy was published in 2014, and numerical aberrations were reported in $40 \%$ of cases. Most cases were diploid ( $n=251,56 \%$ ), whereas $18 \%$ of patients had 47 chromosomes, and $8 \%$ of patients had $43-45$ chromosomes. In no case were less than 43 chromosomes observed. Furthermore, hyperdiploid cases (MN: 48-65) constituted $11 \%$ of all cases. Hyperdiploidy was associated with median age of two years, female sex, and acute megakaryoblastic leukaemia (FAB M7). Among this group only $5 \%$ constituted FAB M2. Gains of chromosomes, in particular 8 (41\%), 21 (19\%), 19 (18\%) and $6(14 \%)$, were the most frequent in children with AML. In contrast, hypodiploid AML ( $8 \%$ of all cases) was associated with older age, male sex, FAB M2, and $t(8 ; 21)(\mathrm{q} 22 ; \mathrm{q} 22)$ with loss of sex chromosomes [45]. Down's syndrome is associated with constitutional trisomy 21 ; however, only $10 \%$ of patients with de novo AML have this abnormality. AML patients with Down syndrome show typical morphology of acute megakaryoblastic leukaemia (M7) and can be cured with standard therapy [46].

Tetraploidy or near-tetraploidy (MN: 81 to 103 chromosomes) is a rare finding in AML and in large series of paediatric AMLs it was detected in eight patients, and most frequently gains of chromosome $21(3 \%, n=16), 19$, and 8 (both $2 \%, n=10$ ) were observed $[45,47]$.

Loss of a chromosome is extremely rare in AML. Most commonly, loss of Y chromosomes in males (8\%, $n=30)$ and $\mathrm{X}$ in females $95 \%, n=13$ ) are detected [45]. Shandahl et al. [45] reported that monosomy 7 was present in $2 \%$ of all AMLs $(n=14)$, whereas monosomy 5 was detected in fewer than $1 \%$ of cases $(n=3)$. Among numerical chromosomal aberrations monosomy 7 was a predictor of poor outcome. In some patients these aberrations are preceded by myelodysplastic phase. Better prognosis is observed in patients with $7 \mathrm{q}$ deletions than in those with complete loss of 7 [48].

Generally, no prognostic relation of $\mathrm{MN}$ and patient outcome was detected in paediatric AMLs [45]. Analysis of a large series of de novo AML indicated a higher incidence of immature myeloblastic phenotype in aneuploid patients and high incidence of expression of CD4, TdT, CD19, and CD7 on leukaemic blasts with respect to diploid cases [49]. So far, there are no data on the association of aneuploidy with any specific immunophenotype or prognosis in AML patients. A summary of numeric chromosomal aberrations in AML is presented in Table 2. 
TABLE 2. Numeric chromosomal aberrations in acute myeloid leukaemia

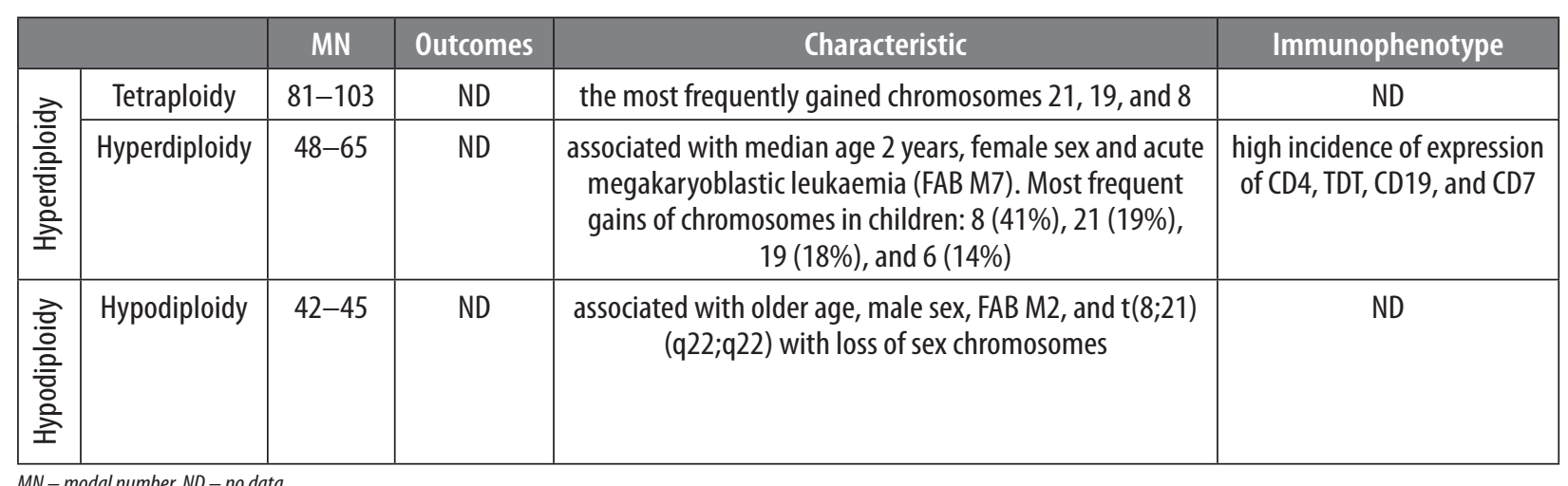

\section{CONCLUSIONS}

Patients with high hyperdiploid karyotype BCP-ALL have a better prognosis than patients with other cytogenetic abnormalities, so they need a less invasive therapy protocol. For this reason, it is reasonable to distinguish between hyperdiploidy and other types of ploidy. Flow cytometry provides rapid detection of abnormal DNA content and can be an alternative tool to conventional cytogenetics at the time of diagnosis. It can also provide additional diagnostically and prognostically useful information before therapy. Because DNA aneuploidy is considered to be an early indicator of cytogenetic abnormality, DI of abnormal cells determined by flow cytometry is suggested to be an additional objective parameter for early detection of cytogenetic abnormality in AL. However, more data are needed about numerical chromosome aberrations and their correlations with immunophenotype, especially through multicentre studies. Further observations are needed in order to use DI as a standard medical procedure. However, it is already an attractive method to improve classification of patients to risk-directed therapy.

\section{DISCLOSURE}

The authors declare no conflict of interest.

\section{REFERENCES}

1. Gilliland DG, Tallman MS. Focus on acute leukemias. Cancer Cell 2002; 1: 417-420.

2. Bennett JM, Catovsky D, Daniel MT, et al. Proposals for the classification of the acute leukaemias. French-American-British (FAB) co-operative group. Br J Haematol 1976; 33: 451-458.

3. Bene MC, Castoldi G, Knapp W, et al. Proposals for the immunological classification of acute leukemias. European Group for the Immunological Characterization of Leukemias (EGIL). Leukemia 1995; 9: 1783-1786.

4. Jaffe ES. The 2008 WHO classification of lymphomas: implications for clinical practice and translational research. Hematology Am Soc Hematol Educ Program 2009: 523-531.
5. Jaffe ES, Barr PM, Smith SM. Understanding the New WHO Classification of Lymphoid Malignancies: Why It's Important and How It Will Affect Practice. Am Soc Clin Oncol Educ Book 2017; 37: 535-546.

6. Vázquez-Reyes A, Bobadilla-Morales L, Barba-Barba C, et al. Aneuploidy identification in pre-B acute lymphoblastic leukemia patients at diagnosis by Multiplex Ligation-dependent Probe Amplification (MLPA). Leuk Res 2017; 59: 117-123.

7. Szczepański T, Harrison CJ, van Dongen JJ. Genetic aberrations in paediatric acute leukaemias and implications for management of patients. Lancet Oncol 2010; 11: 880-889.

8. Wang XM. Advances and issues in flow cytometric detection of immunophenotypic changes and genomic rearrangements in acute pediatric leukemia. Transl Pediatr 2014; 3: 149-155.

9. Ritterbach J, Hiddemann W, Beck JD, et al. Detection of hyperdiploid karyotypes (> 50 chromosomes) in childhood acute lymphoblastic leukemia (ALL) using fluorescence in situ hybridization (FISH). Leukemia 1998; 12: 427-433.

10. Pérez-Vera P, Frías S, Carnevale A, et al. A strategy to detect chromosomal abnormalities in children with acute lymphoblastic leukemia. J Pediatr Hematol Oncol 2004; 26: 294-300.

11. Abad M, Ciudad J, Rincon MR, et al. DNA aneuploidy by flow cytometry is an independent prognostic factor in gastric cancer. Anal Cell Pathol 1998; 16: 223-231.

12. Pinto AE, Pereira T, Silva GL, André S. Prognostic relevance of DNA flow cytometry in breast cancer revisited: The 25-year experience of the Portuguese Institute of Oncology of Lisbon. Oncol Lett 2017; 13: 2027-2033.

13. Kumar BK, Bhatia P, Trehan A, et al. DNA Ploidy and S-phase Fraction Analysis in Paediatric B-cell Acute Lymphoblastic Leukemia Cases: a Tertiary Care Centre Experience. Asian Pac J Cancer Prev 2015; 16: 7917-7922.

14. Rachieru-Sourisseau P, Baranger L, Dastugue N, et al. DNA Index in childhood acute lymphoblastic leukaemia: a karyotypic method to validate the flow cytometric measurement. Int J Lab Hematol 2010; 32: 288-298.

15. Forestier E, Holmgren G, Roos G. Flow cytometric DNA index and karyotype in childhood lymphoblastic leukemia. Anal Cell Pathol 1998; 17: 145-156.

16. Kenney B, Zieske A, Rinder H, Smith B. DNA ploidy analysis as an adjunct for the detection of relapse in B-lineage acute lymphoblastic leukemia. Leuk Lymphoma 2008; 49: 42-48.

17. Harrison C. New genetics and diagnosis of childhood B-cell precursor acute lymphoblastic leukemia. Pediatr Rep 2011; 3 (Suppl 2): e4.

18. Pieters R, Schrappe M, De Lorenzo P, et al. A treatment protocol for infants younger than 1 year with acute lymphoblastic leukaemia (Interfant-99): an observational study and a multicentre randomised trial. Lancet 2007; 370: 240-250. 
19. Nachman JB, Heerema NA, Sather H, et al. Outcome of treatment in children with hypodiploid acute lymphoblastic leukemia. Blood 2007; 110: 1112-1115.

20. Raimondi SC. Cytogenetics in acute leukemias. In: Childhood Leukemias, Pui CH (ed.). Cambrige University Press, New York 2006: 238-265.

21. Pui CH, Carroll AJ, Head D, et al. Near-triploid and near-tetraploid acute lymphoblastic leukemia of childhood. Blood 1990; 76: 590-596.

22. Paulsson K, Forestier E, Lilljebjörn H, et al. Genetic landscape of high hyperdiploid childhood acute lymphoblastic leukemia. Proc Natl Acad Sci U S A 2010; 107: 21719-21724.

23. Paulsson K, Johansson B. High hyperdiploid childhood acute lymphoblastic leukemia. Genes Chromosomes Cancer 2009; 48: 637-660.

24. Zaliova M, Hovorkova L, Vaskova M, et al. Slower early response to treatment and distinct expression profile of childhood high hyperdiploid acute lymphoblastic leukaemia with DNA index $<1.16$. Genes Chromosomes Cancer 2016; 55: 727-737.

25. Moorman AV, Richards SM, Martineau M, et al. Outcome heterogeneity in childhood high-hyperdiploid acute lymphoblastic leukemia. Blood 2003; 102: 2756-2762.

26. Sutcliffe MJ, Shuster JJ, Sather HN, et al. High concordance from independent studies by the Children's Cancer Group (CCG) and Pediatric Oncology Group (POG) associating favorable prognosis with combined trisomies 4, 10, and 17 in children with NCI Standard-Risk B-precursor Acute Lymphoblastic Leukemia: a Children's Oncology Group (COG) initiative. Leukemia 2005; 19: 734-740.

27. Woodward EL, Olsson ML, Johansson B, Paulsson K. Allelic variants of PRDM9 associated with high hyperdiploid childhood acute lymphoblastic leukaemia. Br J Haematol 2014; 166: 947-949.

28. Lustosa de Sousa DW, de Almeida Ferreira FV, Cavalcante Félix FH, de Oliveira Lopes MV. Acute lymphoblastic leukemia in children and adolescents: prognostic factors and analysis of survival. Rev Bras Hematol Hemoter 2015; 37: 223-229.

29. Raimondi SC, Roberson PK, Pui CH, et al. Hyperdiploid (47-50) acute lymphoblastic leukemia in children. Blood 1992; 79: 3245-3252.

30. Hrusák O, Porwit-MacDonald A. Antigen expression patterns reflecting genotype of acute leukemias. Leukemia 2002; 16: 1233 1258.

31. Djokic M, Björklund E, Blennow E, et al. Overexpression of CD123 correlates with the hyperdiploid genotype in acute lymphoblastic leukemia. Haematologica 2009; 94: 1016-1019.

32. Muñoz L, Nomdedéu JF, López O, et al. Interleukin-3 receptor alpha chain (CD123) is widely expressed in hematologic malignancies. Haematologica 2001; 86: 1261-1269.

33. Harrison CJ, Moorman AV, Broadfield ZJ, et al. Three distinct subgroups of hypodiploidy in acute lymphoblastic leukaemia. Br J Haematol 2004; 125: 552-559.

34. Safavi S, Olsson L, Biloglav A, et al. Genetic and epigenetic characterization of hypodiploid acute lymphoblastic leukemia. Oncotarget 2015; 6: 42793-42802.

35. Raimondi SC, Zhou Y, Mathew S, et al. Reassessment of the prognostic significance of hypodiploidy in pediatric patients with acute lymphoblastic leukemia. Cancer 2003; 98: 2715-2722.

36. Heerema NA, Nachman JB, Sather HN, et al. Hypodiploidy with less than 45 chromosomes confers adverse risk in childhood acute lymphoblastic leukemia: a report from the children's cancer group. Blood 1999; 94: 4036-4045.

37. Holmfeldt L, Wei L, Diaz-Flores E, et al. The genomic landscape of hypodiploid acute lymphoblastic leukemia. Nat Genet 2013; 45 : 242-252.
38. Safavi S, Paulsson K. Near-haploid and low-hypodiploid acute lymphoblastic leukemia: two distinct subtypes with consistently poor prognosis. Blood 2017; 129: 420-423.

39. Gibbons B, MacCallum P, Watts E, et al. Near haploid acute lymphoblastic leukemia: seven new cases and a review of the literature. Leukemia 1991; 5: 738-743.

40. Chessels JM, Swansbury GJ, Reeves B, et al. Cytogenetics and prognosis in childhood lymphoblastic leukaemia: results of MRC UKALL X. Medical Research Council Working Party in Childhood Leukaemia. Br J Haematol 1997; 99: 93-100.

41. Park J, Kim M, Lee HK, et al. Chromosome abnormalities in T-cell acute lymphoblastic leukemia in Korea. Int J Hematol 2014; 99: 279-287.

42. Garcia DR, Bhatt S, Manvelyan M, et al. An unusual T-cell childhood acute lymphoblastic leukemia harboring a yet unreported near-tetraploid karyotype. Mol Cytogenet 2011; 4: 20.

43. Lemez P, Attarbaschi A, Béné MC, et al. Childhood near-tetraploid acute lymphoblastic leukemia: an EGIL study on 36 cases. Eur J Haematol 2010; 85: 300-308.

44. Lazarevic V, Rosso A, Juliusson G, et al. Prognostic significance of high hyperdiploid and triploid/tetraploid adult acute myeloid leukemia. Am J Hematol 2015; 90: 800-805.

45. Sandahl JD, Kjeldsen E, Abrahamsson J, et al. Ploidy and clinical characteristics of childhood acute myeloid leukemia: A NOPHO-AML study. Genes Chromosomes Cancer 2014; 53: 667-675.

46. Creutzig U, Reinhardt D, Diekamp S, et al. AML patients with Down syndrome have a high cure rate with AML-BFM therapy with reduced dose intensity. Leukemia 2005; 19: 1355-1360.

47. Huang L, Wang SA, DiNardo C, et al. Tetraploidy/near-tetraploidy acute myeloid leukemia. Leuk Res 2017; 53: 20-27.

48. Hasle H, Alonzo TA, Auvrignon A, et al. Monosomy 7 and deletion $7 \mathrm{q}$ in children and adolescents with acute myeloid leukemia: an international retrospective study. Blood 2007; 109: 4641-4647.

49. Vidriales MB, Orfao A, López-Berges MC, et al. DNA aneuploidy in acute myeloblastic leukemia is associated with a high expression of lymphoid markers. Cytometry 1995; 22: 22-25. 